\title{
The Himalayan Onion (Allium wallichii Kunth) Harbors Unique Spatially Organized Bacterial Communities
}

\author{
Xiaoyulong Chen ${ }^{1,2,3,4} \cdot$ Lisa $\mathrm{Krug}^{5} \cdot$ Maofa Yang $^{2} \cdot$ Gabriele Berg $^{5} \cdot$ Tomislav Cernava $^{2,5}$ (D)
}

Received: 19 October 2020 / Accepted: 1 March 2021 / Published online: 16 March 2021

(C) The Author(s) 2021

\begin{abstract}
Plant-associated microorganisms are known to contribute with various beneficial functions to the health and productivity of their hosts, yet the microbiome of most plants remains unexplored. This especially applies to wild relatives of cultivated plants, which might harbor beneficial microorganisms that were lost during intensive breeding. We studied bacterial communities of the Himalayan onion (Allium wallichii Kunth), a wild relative of onion native to mountains in East Asia. The bacterial community structure was assessed in different plant microhabitats (rhizosphere, endosphere, anthosphere) by sequencing of 16S rRNA gene fragment amplicons. Targeted bioinformatic analyses were implemented in order to identify unique features in each habitat and to map the overall community in the first representative of the Amaryllidaceae plant family. The highest bacterial diversity was found for bulk soil (Shannon index, $H^{\prime}$ 9.3) at the high-altitude sampling location. It was followed by the plant rhizosphere $\left(H^{\prime}\right.$ 8.9) while communities colonizing flowers $\left(H^{\prime} 6.1\right)$ and the endosphere $\left(H^{\prime} 6.5\right.$ and 5.6) where less diverse. Interestingly, we observed a non-significant rhizosphere effect. Another specificity of the microbiome was its high evenness in taxonomic distribution, which was so far not observed in plant microbiomes. Pseudomonas was identified among additional 10 bacterial genera as a plant-specific signature. The first insights into the microbiome of a plant in the widespread Allium genus will facilitate upcoming comparisons with its domesticated relatives while additionally providing a detailed microbiome mapping of the plant's microhabitats to facilitate bioresource mining.
\end{abstract}

Keywords Plant microbiome $\cdot$ Bacterial communities $\cdot$ Endophytic bacteria $\cdot$ Rhizosphere $\cdot$ Phyllosphere

\section{Introduction}

Various members of the Amaryllidaceae plant family find application in traditional as well as modern cuisine. They are often characterized by rich secondary metabolite profiles, which can include sulfur-containing compounds with a

Tomislav Cernava

tomislav.cernava@tugraz.at

1 Key Laboratory of Green Pesticide and Agricultural Bioengineering, Ministry of Education, Guizhou University, Guiyang 550025, China

2 College of Tobacco Science, Guizhou University, Guiyang 550025, China

3 Key Laboratory of Agricultural Microbiology, Guizhou University, Guiyang 550025, China

4 College of Science, Tibet University, Lhasa 850012, China

5 Institute of Environmental Biotechnology, Graz University of Technology, 8010 Graz, Austria characteristic smell [1]. Globally, the most relevant members are constituted by various cultivars of the common onion (Allium cepa L.) and garlic (Allium sativum L.). The direct ancestor of onions is likely extinct; however, most of the recent wild relatives originate from Asia. Similar to their cultivated relatives, distinct wild species of the Amaryllidaceae also find application as ingredients of traditional dishes as well as sources of bioactive compounds in phytomedicine [2-4]. This includes the Himalayan onion Allium wallichii Kunth, a perennial herb with a single, conical, and short bulb, which is native to India, Nepal, Bhutan, Myanmar, and Southwestern China (Guangxi, Guizhou, Hunan, Sichuan, Tibet, and Yunnan provinces). The anatomy of the plant is reminiscent of the spring onion (Allium fistulosum L.), and it often occurs in natural monocultures. Another characteristic of the plant is that it grows at elevations of 2300 to $4800 \mathrm{~m}$ [5]. Jiucaiping Mountain, with an altitude of $2900 \mathrm{~m}$ above sea level, is the highest point of Guizhou Province, China, and harbors the largest known habitat (approx. 700 ha) of wild A. wallichii 
in the Yunnan-Guizhou Plateau [6]. The mountain is known for its unique landscape during flowering season of A. wallichii in August and September. The extracts from the Himalayan onion were shown to be promising candidates for medicinal applications due to anti-cancer activity and low cytotoxicity [7]. In addition, the extracts exerted anti-microbial activity against known pathogens including Bacillus cereus, Escherichia coli, and Pseudomonas aeruginosa [8]. However, so far, nothing is known about the microbiome found in natural vegetation predominantly covered by $A$. wallichii and microbial communities colonizing the plant itself.

During the last years, the plant microbiota was shown to play a crucial role for host health, protection against phytopathogens, and resistance towards abiotic stresses [9, 10]. Plants were shown to acquire microorganisms from the surrounding environment, but can also equip their seeds with beneficial endophytes $[11,12]$. In the plant rhizosphere, rhizobacteria and mycorrhizal fungi can assist plants against drought stress and contribute to the nutrition by nitrogen fixation [13] as well as increase the availability of phosphorus [14] and potassium [15]. Additionally, beneficial microbes that naturally occur in association with distinct plants can be used as a resource for novel biocontrol and plant growth promotion applications in other vegetation systems $[16,17]$. In addition to commonly exploited microorganisms from soil, the plant rhizosphere and endorhiza, microorganism from aboveground tissues, including stem and flower (anthosphere), are also promising candidates for biotechnological applications $[18,19]$. Therefore, in order to provide a complete map of a plant's microbial community, it is important to include all major tissue types in the analysis. Bacteria show specificity not only for plant tissues but also for plant genotypes $[20,21]$. In this context, it was shown that plants with bioactive secondary metabolites usually harbor highly specific microbial communities that are shaped by those substances [22-24]. Less is known about the bacterial component within the microbiome of the Amaryllidaceae plant family, while its mycorrhiza was already intensively studied [25]. Moreover, the microbiome of stored onions (Allium cepa) was recently explored by Yurgel and colleagues [26]; they identified Pseudomonas as a biomarker for healthy onions.

Our objective was to analyze the microbiome of Allium wallichii grown in their native environment to provide a first basis to understand and manage the microbiome of our oldest and still widely used crops: onion and garlic. Due to the unique karst landscape and large-scale natural monoculture, we hypothesized that Allium wallichii plants growing on the Jiucaiping Mountain, which is a central part of the YunnanGuizhou Plateau, are colonized by uniquely composed, spatially organized bacterial communities in its different tissues. Pseudomonas, the biomarker for healthy onions [26], was expected to be a prevalent colonizer of their wild relative. Therefore, we explored the microbiome of Allium wallichii by amplicon-based $16 \mathrm{~S}$ rRNA gene sequencing, in order to make it available as a novel bioresource for upcoming biotechnological applications.

\section{Material and Methods}

\section{Sample Collection and Total Community DNA Extraction}

All samples were obtained from a large, natural A. wallichii monoculture growing on Jiucaiping Mountain in Guizhou

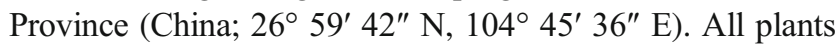
were unearthed during the flowering stage and stored on ice in sterile zip bags until their arrival in the laboratory. Soil samples that were used for complementary analyses were samples in a radial distance of $20-30 \mathrm{~cm}$ from the plants. First, the top soil was removed, then root-free material was obtained from a depth of $10 \mathrm{~cm}$ and immediately manually homogenized inside sterile zip bags to remove macro-aggregates. For soil samples, four replicates were obtained, while for the rhizosphere samples and each plant tissue type, six replicates were obtained, respectively. The plant samples were further processed in the laboratory by separating different plant tissues with sterile scalpels. Rhizosphere samples were obtained by washing adhering soil from roots in $20 \mathrm{ml}$ of $0.85 \% \mathrm{NaCl}$ at $120 \mathrm{rpm}$ for $10 \mathrm{~min}$. The suspension was aliquoted into $2-\mathrm{ml}$ reaction tubes and centrifuged at $14,000 \times \mathrm{g}$ for $20 \mathrm{~min}$ to obtain pellets for further processing. Samples of the endorhiza and the pedicule endosphere were obtained by surface sterilizing 3-5-cm-long segments of each sample type. The segments were first submerged in $4 \%$ sodium hypochlorite $(\mathrm{NaClO})$ solution and placed on a shaker $(120 \mathrm{rpm})$ for 5 min. Subsequently, the segments were rinsed with sterile $\mathrm{ddH}_{2} \mathrm{O}$ and then washed in sterile reaction tubes with sterile $\mathrm{ddH}_{2} \mathrm{O}$ on a shaker $(120 \mathrm{rpm}$ ) for $5 \mathrm{~min}$. The washing step was repeated for two times in order to remove residual $\mathrm{NaClO}$ from the samples. Flowers were directly removed from the plant under sterile condition and subjected to homogenization without further treatments. Subsequently, the plant samples were treated with an automated tissue homogenizer (BBI Life Sciences; Shanghai, China). The homogenates as well as the soil and rhizosphere samples were transferred into extraction tubes under sterile conditions. The total community DNA was extracted from all samples with the FastDNA SPIN Kit for Soil (MP Biomedicals, USA) following the manufacturer's protocol. Subsequently samples were analyzed with NanoDrop (Thermo Fisher Scientific, USA) to confirm the DNA recovery.

\section{Amplification of 16S rRNA Gene Fragments}

The total community DNA extracts from six replicates per sample type were used for PCR-based amplifications with 
the primers 515f (5' GTGYCAGCMGCCGCGGTAA) and 806r (5' GGACTACHVGGGTWTCTAAT) according to the Earth Microbiome Project protocol (www. earthmicrobiome.org) [27] with sample-specific barcodes and Illumina sequencing adaptors. In addition, specific peptide nucleic acid (PNA) oligomers were added to the PCR mix to prevent the amplification of mitochondrial (mPNA) or plastidial (pPNA) RNA from eukaryotes [28]. The resulting amplicons were used to construct a next-generation sequencing library suitable for sequencing on an Illumina PE250 platform and processed with a targeted output of $\geq 100,000$ reads per sample (Novogene; Beijing, China).

\section{Bioinformatic Analyses}

The data was subjected to a standardized workflow for further quality filtering and dereplication. Paired end reads obtained from an Illumina PE250 instrument (paired-end reads; 250-bp read length) were assigned to samples based on their unique barcode sequence and truncated by removing barcode and primer sequences by the sequencing company (Novogene; Beijing, China). Demultiplexed paired end reads were imported in QIIME 2 (2018.11) and further subjected to quality filtering. By applying the dada2 algorithm, paired-end sequences were denoised and dereplicated and chimeras were removed. Taxonomic analysis of amplicon sequencing variants (ASVs) was based on a Naïve-Bayes classifier that was trained on the SILVA 128 release database with clustering at 99\% similarity [29]. The generated feature table (Table S1) was used for all subsequent analyses. After removing mitochondrial, chimeric, and plastid sequences, the 16S rRNA dataset was normalized to 1677 reads per sample to account a variation in the samples reaching from a maximum of 616,087 to a minimum of 1677 reads. Following rarefication of the feature table, alpha and beta diversity analyses were conducted in combination with complementary statistics using QIIME 2 core diversity metrics. Principal coordinate analysis (PCoA) plots were constructed on the basis of an unweighted UniFrac distance matrix that is implemented in QIIME 2. Phylogenetic metrics were generated by aligning representative sequences using the mafft program. After masking the multiple sequence alignment, a phylogenetic tree was generated with FastTree. Statistics were calculated by applying analyses of similarity (ANOSIM) within the QIIME 2 pipeline for identifying significant differences in beta diversity between groups. The alpha diversity was assessed through observed OTU and Shannon diversity indices and statistics calculated by applying Kruskal-Wallis tests including Benjamini/Hochberg FDR adjustment within QIIME 2. Significantly different abundant features were identified by applying the Kruskal-Wallis test including FDR multiple test correction within QIIME 1.9.0. As input for calculations, a non-rarefied feature table excluding mitochondrial and plastid sequences was used. Differences in evenness were calculated using one-way ANOVA including Bonferroni multiple test correction in IBM SPSS (version 25.0; IBM Corporation, NY, USA).

For constructing the OTU network, the feature table was reduced by retaining only features which occur in $\geq 75 \%$ of the replicates in each group (further referred to as core microbiota) and with a mean relative abundance of at least $0.1 \%$ in the whole dataset. The OTU network was constructed using the QIIME 1.9.0 pipeline (make_otu_network.py) and visualized using Cytoscape version 3.7.0. For generating bar charts, features were collapsed on genus level and a reduced table containing only taxa with a mean relative abundance of at least $0.5 \%$ in the whole dataset served as input. Bar charts represent mean relative abundance of each group. A collapsed feature table (genus level) served also as input for the generation of the phylogenetic tree including all taxa with a mean relative abundance of at least $0.05 \%$ within the whole dataset. A phylogenetic tree was constructed with extracted Pseudomonas ASVs by using MEGA X (version 10.0.4) and the implemented neighbor-joining method with 1000 bootstraps.

\section{Physicochemical Soil Analyses}

Bulk soil was obtained at the same sampling location as A. wallichii and collected at a depth of $10 \mathrm{~cm}$, which corresponds to the approximate root depth of the plants. A total of $500-\mathrm{g}$ soil was obtained and characterized in terms of $\mathrm{pH}$ level, organic matter fraction, total nitrogen content, total phosphorous content, total potassium, total water-soluble salts, and cation exchange capacity by the service provider United Nation Quality Detection Co., Ltd. (Xi'an, Shaanxi, China).

\section{Results}

\section{Diversity Analyses}

After filtering chimeric, mitochondrial, and plastid sequences, feature table was reduced from a total read count of $8,925,788$ to $3,380,349$ sequences representing 14,140 features. Highly abundant features that remained without classification at lower taxonomic levels were manually blasted against the NCBI nt_collection; results indicated that several of those features were plastid or chimeric sequences and were therefore excluded prior to further analyses. The filtered feature table contained 3,250,046 sequences representing 13,361 features. Following normalization of the feature table to 1677 reads per sample, 5266 features remained. These features were collapsed on genus level resulting in 992 taxa. Alpha rarefaction analyses revealed the highest Shannon index for soil $\left(H^{\prime}=9.34 \pm\right.$ 
$0.10)$ followed by the rhizosphere $\left(H^{\prime}=8.87 \pm 0.46\right)$. Shannon diversity indices for flower, pedicel, and endorhiza were calculated as $6.11 \pm 0.80,6.50 \pm 0.44$, and $5.59 \pm 0.93$, respectively, whereas no significant differences in alpha diversity were observed within these plant-associated microhabitats $(p>0.05)$. A detailed breakdown of Shannon indices and complementary statistics is shown in Table S2. The same results were obtained when assessing bacterial diversity based on observed OTUs. The highest number of observed OTUs was revealed for soil $(950 \pm 24)$ followed by the rhizosphere $(822 \pm 107)$. Flower $(195 \pm 45)$, pedicel $(204 \pm 31)$, and endorhiza $(146 \pm 49)$ shared similar numbers of observed OTUs with no significant differences (Figure S1; Table S3). Beta diversity was assessed by calculating the unweighted UniFrac distance matrix, and statistics were calculated using pairwise ANOSIM. Distinct clustering of plant-associated microhabitats (flower, pedicel, endorhiza) and soil-associated habitats (rhizosphere and soil) was observed, reflecting the statistical analyses; within the plant (flower, pedicel, endorhiza), no significant $(p>0.05)$ differences in community compositions were calculated; the same observation was made when differences in beta diversity between rhizosphere and soil were assessed (Fig. 1; Table S4). Community compositions differed significantly when pairwise comparisons were conducted between each plant microhabitat and either soil or the rhizosphere. All samples showed a very high evenness whereas bacterial features are most evenly distributed within the soil $(E=$ $0.944 \pm 0.010)$ followed by the rhizosphere $(E=0.917 \pm$ 0.030 ). Significant differences in evenness were found between the endorhiza $(E=0.783 \pm 0.088)$ and soil as well as between the endorhiza and the rhizosphere, respectively (Fig. 2; Table S5).

\section{Bacterial Community Structure in Different Plant Microhabitats}

Overall, the normalized feature table contained 42 different phyla; among them, Proteobacteria were predominating in the rhizosphere (39\%) followed by Bacteroidetes (14\%), Acidobacteria (12\%), Actinobacteria (7\%), Firmicutes (6\%), Chloroflexi (6\%), Planctomycetes (6\%), Verrucomicrobia (5\%), and Gemmatimonadetes (1\%). Within the soil, the dominating phyla were Proteobacteria (28\%) followed by Acidobacteria (17\%) and Bacteroidetes (11\%), Actinobacteria (9\%), Planctomycetes (8\%), Chloroflexi (7\%), Verrucomicrobia (6\%), Firmicutes $(5 \%)$, and Gemmatimonadetes (1\%); with 5\% relative abundance, Archaea were most abundant in soil while in all other samples, their relative abundance was less than $1 \%$. In the flower, the most abundant bacterial phyla were Proteobacteria (51\%), followed by Firmicutes (20\%), Bacteroidetes (15\%), Actinobacteria (7\%), Deinococcus-Thermus (1\%), and
Chloroflexi (1\%). The pedicel was dominated by Firmicutes (40\%) followed by Proteobacteria (33\%), Bacteroidetes (13\%), and Actinobacteria (8\%). In the endorhiza, the most abundant phyla were Proteobacteria (47\%) followed by Firmicutes (19\%), Actinobacteria (18\%), Chloroflexi (2\%), and Acidobacteria (2\%). Phyla with a relative abundance of less than $0.5 \%$ were not included in deepening assessments (Fig. 3; Table S6). In order to detect habitat-specific occurrence of distinct bacterial features, a statistical assessment of differential abundance was conducted. Within the plant tissues (flower, pedicel, endorhiza), no significantly different abundant features were identified. The same observation was made when soil and rhizosphere were compared. However, when plant tissue-associated taxa were contrasted to such occurring in the other microhabitats (group A: flower, pedicel, endorhiza; group B: rhizosphere, soil), 3595 of 13,361 features (27\%) were significantly different abundant in the compared groups. Of those 3595 features, only 11 were unique for the plant tissues (Nocardiopsis, Hymenobacter, Pseudomonas, Aureimonas, Clostridium, Macellibacteroides, Bacteroides, not further classified Verrucomicrobiaceae, not further classified Microbacteriaceae, Micrococcus, Phascolartobacterium), while 3369 were unique in the rhizosphere/soil group. In total, 215 features were found in both groups, but showed significantly different abundances therein. Only seven of those 215 features were significantly more abundant in the plant than in the rhizosphere/soil. Those features were assigned to the bacterial genera Streptococcus, Mycobacterium, Bacteroides, Subdoligranulum, not further classified Lachnospiraceae, and not further classified Ruminococcaceae.

\section{Habitat-Specific Microbial Signatures}

A phylogenetic tree, which was constructed on the basis of prevalent members of the microbiome, included 269 taxa with a mean relative abundance of $0.05 \%$ over the whole dataset (Fig. 4). For the flower, the pedicel, and the endorhiza, 44, 62, and 29 bacterial taxa were identified to occur in their respective core microbiota. The rhizosphere harbored 131 core taxa and the soil 177 . Overall, only seven bacterial taxa occurred in the core microbiota of all analyzed microhabitats (Pseudomonas, Sphingomonas, not further classified Comamonadaceae, Bacteroides, not further classified Bacteroidales, Acinetobacter, and Duganella). Five core taxa were identified to be unique in plant microhabitats (flower, pedicel, endorhiza) including Streptococcus, Nocardiopsis, Prevotella, Corynebacterium, and Propionibacterium, while soil and rhizosphere shared in total 98 core taxa. Only one unique core taxon was identified for the flower (Macellibacteroides) while 19 taxa occurred only in the core microbiota of the pedicel and four taxa were identified as unique core taxa for the endorhiza (not further classified Planococcaceae, Romboutsia, Tumebacillus, 

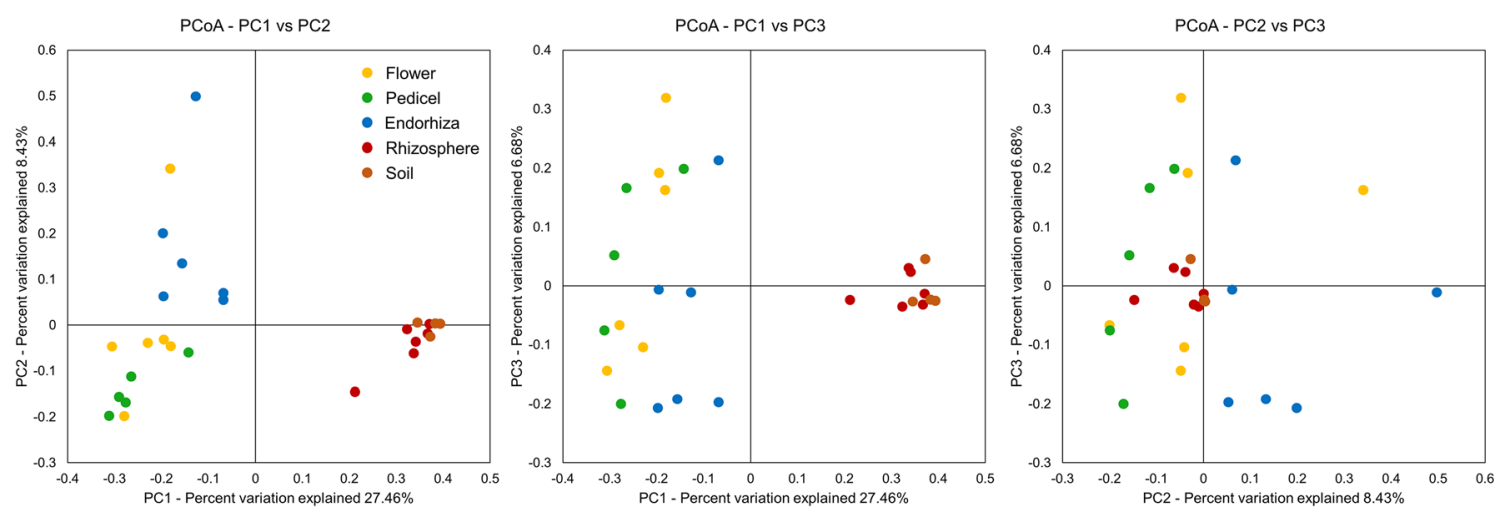

Fig. 1 Beta diversity assessment in different plant tissues, the rhizosphere, and surrounding soil. A principal coordinate analysis (PCoA) was conducted to visualize community structure similarity and clustering between different sample types. The percentages of the three dimensions, which explain the highest degree of variance, were included

in the visualization. Different colors of the dots indicate distinct sample types in the plant microbiome and the bulk soil. Complementary statistical analyses were used to assess the significance of dissimilarity between samples and summarized in Table S4

Coxiella). The soil harbored 40 unique core taxa, while for the rhizosphere, no unique core taxa were identified. Due to the prominent occurrence of Pseudomonas in plant-associated microhabitats, a detailed analysis was conducted with all representatives that were recoverable from the amplicon library (Figure S2). A genus-specific phylogenetic tree that was constructed with the recovered Pseudomonas ASVs and available reference sequences indicated the presence of potentially novel lineages that showed low sequence similarity to available isolates.

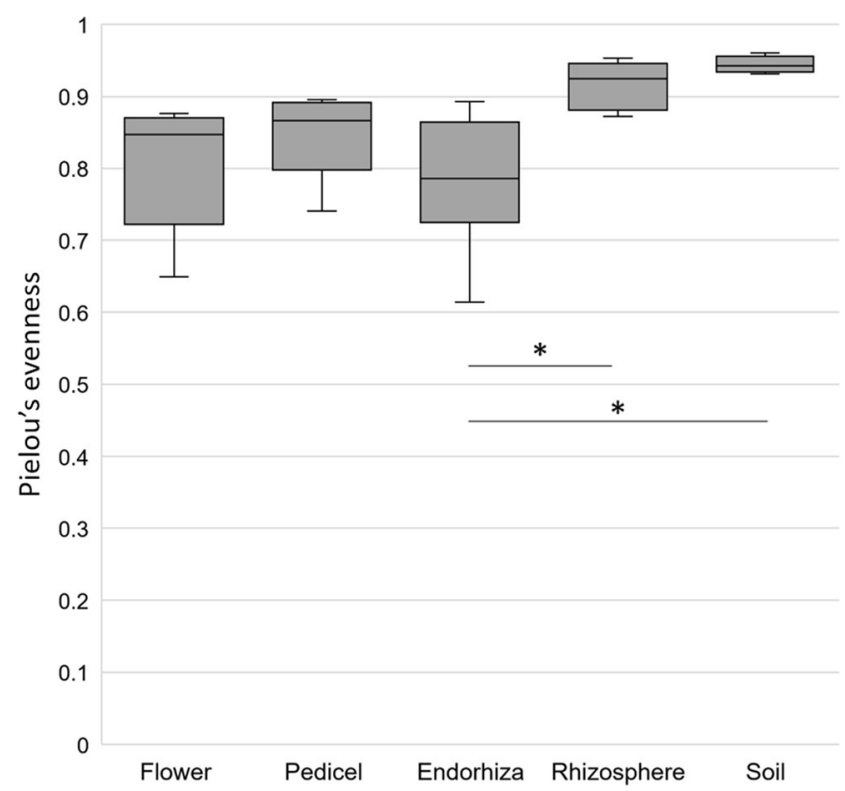

Fig. 2 Evenness of the plant-associated bacterial communities and bulk soil. The dataset was subjected to Pielou's evenness analysis in order to obtain a numeric measure for the evenness that was evident from the community structures across different sample types. Significant differences in evenness were calculated by pairwise comparison using ANOVA including Banferroni multiple test correction and summarized in Table S5

\section{Assessment of Connectivity Between Plant Microhabitats}

The connectivity between all analyzed microhabitats was analyzed by constructing a feature-level network. All features were visualized as nodes and connected with edges to the respective microhabitat of the plant where they were detected. In this analysis, which is based on a higher taxonomic resolution than the genus-level profiling, most features were found to be shared between the rhizosphere and the soil (76 features), including 43 features assigned to Proteobacteria, 12 features assigned to Acidobacteria, six to Bacteroidetes, five features assigned to Actinobacteria, four to Chloroflexi, three to Firmicutes, two to Verrucomicrobia, and one to Gemmatimonadetes. The second highest number of features (20) was shared between the flower and the pedicel with 12 features assigned to Firmicutes, five features to Proteobacteria, two to Bacteroidetes, and one to Actinobacteria. While the flower harbored no unique features, the most unique features (15) were identified for the pedicel including seven features assigned to Firmicutes, four to Bacteroidetes, two to Actinobacteria, and two assigned to Proteobacteria. The endorhiza, the rhizosphere, and the soil harbored six, 10, and 13 unique features, respectively. Only two features were shared by all microhabitats; they were assigned to Pseudomonas and Duganella. In addition, it was found that one feature assigned to Bacillales was shared between the endorhiza and the rhizosphere (Fig. 5).

\section{Soil Physicochemical Parameters}

The sampled soil from the A. wallichii field on Jiucaiping Mountain is typical clay loam. It had a close to neutral $\mathrm{pH}$ of 6.81 , a cation exchange capacity of $12.2 \mathrm{cmol} / \mathrm{kg}$, and a medium salinity with $8.5 \mathrm{~g} / \mathrm{kg}$ water-soluble salts. In terms of 


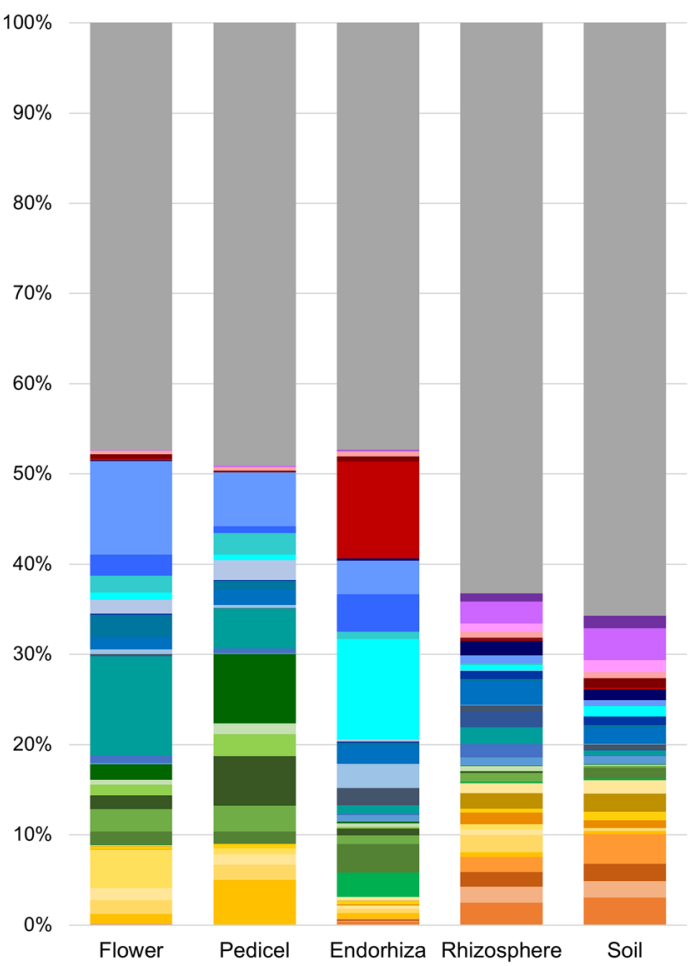

Fig. 3 Bacterial community structure in the A. wallichii microbiome. The bacterial community structure was visualized up to genus level for each sample type. When genus-level assessment was not possible, the taxonomic information was provided on family, order, or phylum level and

plant nutrients, the total nitrogen content was $1.31 \%$, the organic matter fraction $1.48 \%$, the total phosphorus $601 \mathrm{mg} / \mathrm{kg}$, and the total potassium concentration $114.24 \mathrm{mg} / \mathrm{kg}$.

\section{Discussion}

The present study provides a detailed profiling of a native plant to Eastern Asia that is of local importance in Southwestern China and which might be a biotechnologically relevant resource in the future. The Allium wallichii microbiome was characterized by a high degree of microhabitat specificity especially between the aboveground and belowground parts, a missing rhizosphere effect, an unusual evenness within the bacterial community structure, and Pseudomonas-rich hotspots. Pseudomonas was identified as a biomarker for healthy onions [26], but is also a model for beneficial, plant-associated bacteria for a long time [30, 31]. Further exploration of the natural Pseudomonas population associated with Himalayan onion is a promising strategy to discover new biological resources for modern agriculture. It might harbor distinct strains that are transferable to its cultivated relatives that are often affected by disease outbreaks. Here, especially the high prevalence of soil-borne phytopathogenic fungi affects the cultivation of onion [32] and thus might profit from new biological solutions.
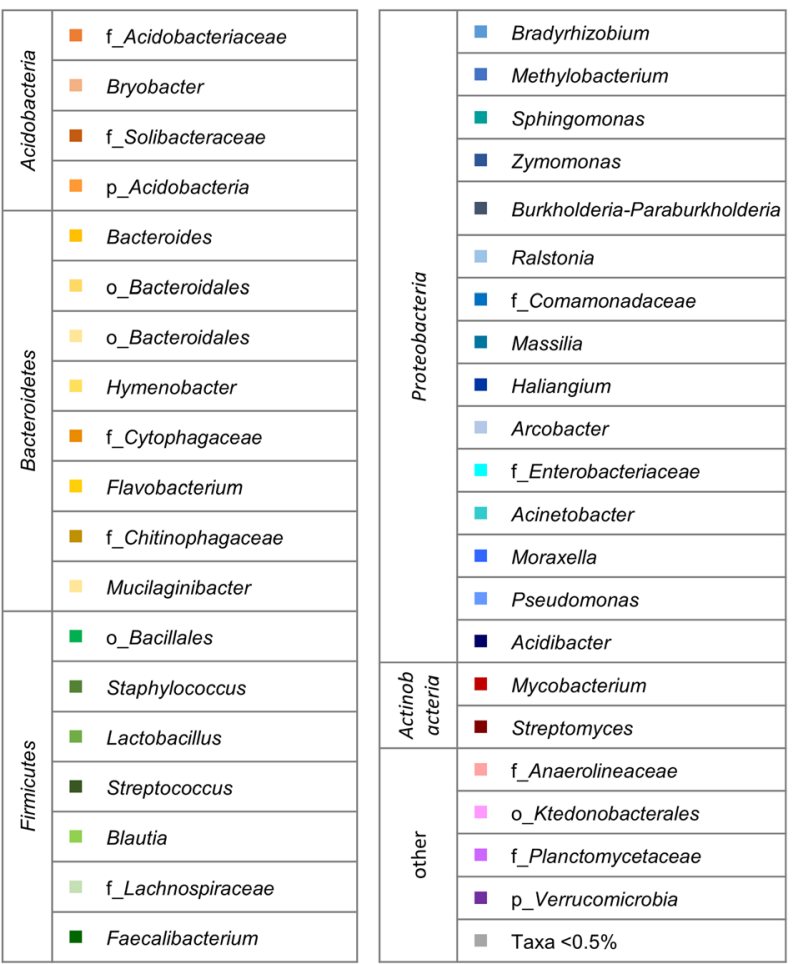

indicated with a prefixed letter. Taxonomic assignments at higher levels are provided and clustered in the legend. All taxa with an occurrence of $\geq$ $0.5 \%$ were included in the legend. Taxa with a lower abundance were summarized as "taxa $<0.5 \%$ "

When the two endosphere habitats (endorhiza and pedicule endosphere) of the plant were compared, their bacterial community compositions showed substantial differences. The endorhiza showed a prevalence of Actinobacteria and Enterobacteriaceae at higher taxonomic levels while the pedicule endosphere was colonized by a high number of Firmicutes. Each of these bacterial groups showed a contrary trend in the respective other endosphere of Allium wallichii. We assume that this distribution is due to differences in physicochemical conditions of the two niches. Nevertheless, Actinobacteria as well as Firmicutes might harbor members that are of potential interest as new bioresources due to their high biocontrol potential $[17,33]$. Interestingly, we have also observed a higher similarity of the bacterial community in the endorhiza to the community found on the plant's flowers when compared with the rhizosphere microbiome. This is an indication for the high selectivity of the plant and a filter effect that was previously also observed with crop plants [11]. Soilborne microorganisms have to either pass through the plant's endosphere to reach aboveground plant parts or they are transferred by repelling raindrops or splashing irrigation water in cultivated plants [34].

We have identified two specificities in the A. wallichii microbiome that differentiates it from most other plants that were subjected to bacterial community analyses in the past years. First, the rhizosphere effect of the plants was low, and 
Fig. 4 Assessment of common bacterial taxa in different tissues of $A$. wallichii. The tree graph includes taxa with a minimum relative abundance of $0.05 \%$ within the whole dataset. All features were collapsed at genus level to reduce the complexity of the visualization. Coloration in the outer rings indicates the occurrence of distinct taxa in the indicated sample types. Node sizes correspond to the mean relative abundance of distinct taxa over the whole dataset; three representative node sizes are provided with the respective percentages

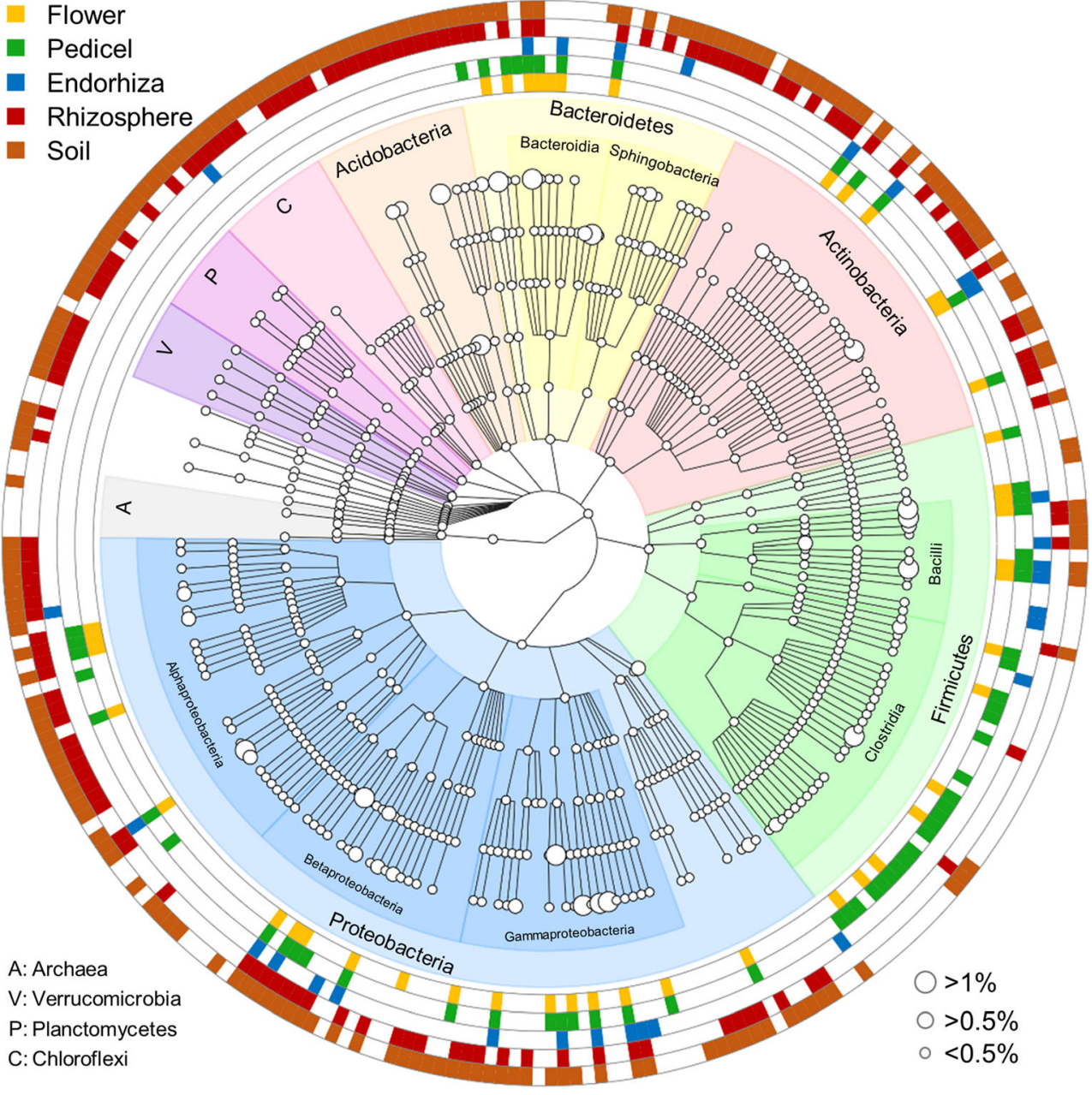

no significant differences were observed when compared with the surrounding soil. The complementary physicochemical soil analyses indicated that the characteristics of the natural monoculture are representative for this region. Previous studies that were conducted in the proximity of Jiucaiping Mountain reported $\mathrm{pH}$ values as well as total nitrogen concentrations that were in the same range $[35,36]$. Plants direct a substantial amount of fixed nitrogen and carbon to the rhizosphere trough specific exudates [37]. These exudates attract and nourish populations of beneficial rhizosphere microorganisms through the plant's whole lifecycle [20,38]. We hypothesize that either the soil in the A. wallichii fields of Jiucaiping Mountain is influenced by the naturally occurring monoculture to a level that led to a homogenization of the rhizosphere and the surrounding soil, or that the plant has a low potential to attract microorganisms from the surrounding soil. The latter might be likely, because A. wallichii has a proportionally small root system that consists of a low number of primary roots. Plant exudates are mainly excreted from lateral roots, which also serve as entry points for nodulating bacteria or other beneficial endophytes [37, 39]. Further indications for the second hypothesis are given by the fact the bulb plants have a specific lifecycle and are often associated with a mycorrhiza [25]. Interestingly, distinct Pseudomonas strains are known due to their positive interactions with certain fungi as "mycorrhiza helper bacteria" [40]. Their high occurrence might be indicative for the same role in the A. wallichii microbiome, which remains to be confirmed. It is also noteworthy to mention that plants were collected during the flowering stage for the current study and that it was previously found that plants show a lower rhizosphere effect at later development stages [41, 42]. Targeted analyses will be required in the future to clarify if this also applies to members of the Amaryllidaceae plant family.

As a second specificity of the A. wallichii microbiome, our dataset was characterized by a high microbial diversity, and more remarkably by an exceptional evenness in the distribution of different taxa. This is indicative for a stable and undisturbed ecosystem and is not commonly observed with cultivated plants. While less attention is paid to community evenness than to diversity and microbial abundance in general [43], it is an important determinant for resilience of various 


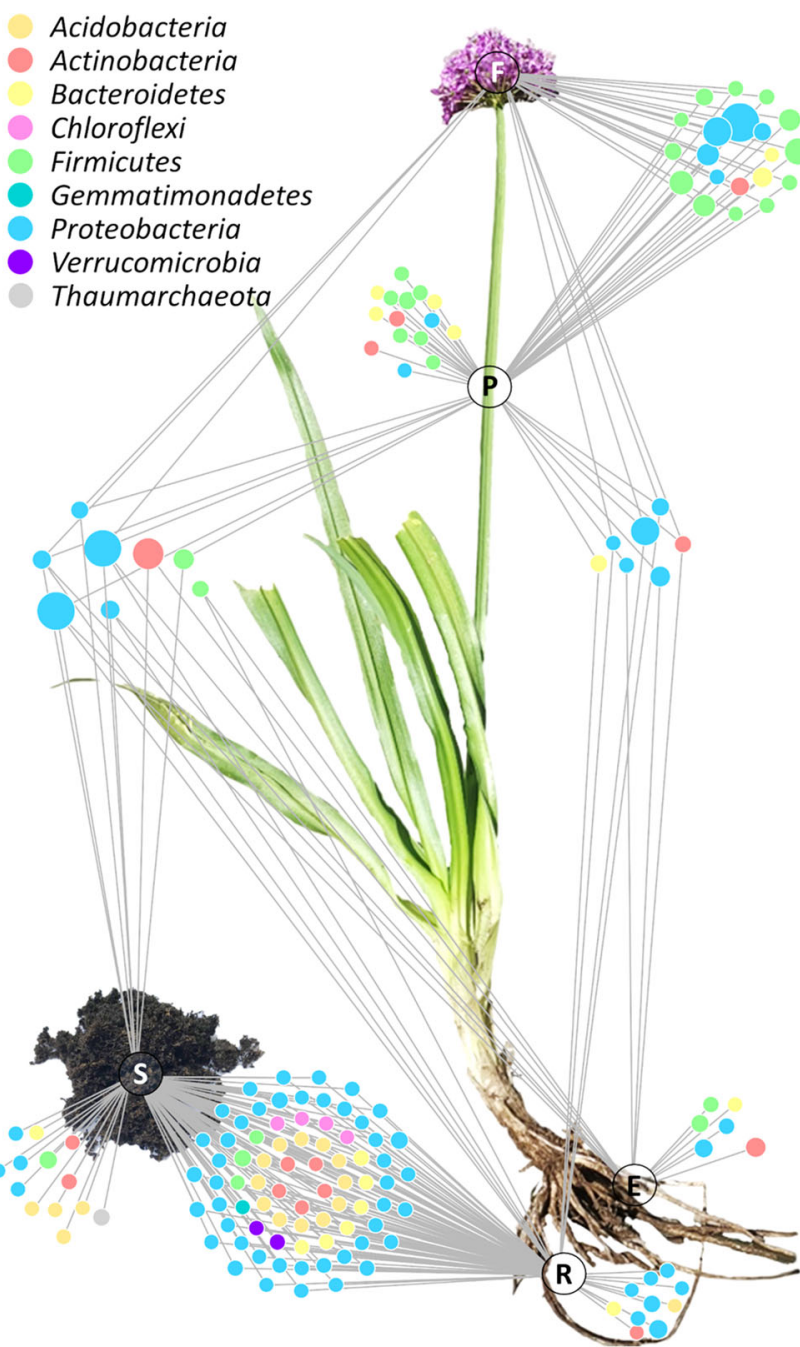

Fig. 5 Identification of common bacterial components across plant habitats at feature level. The network was rendered with Cytoscape 3.7.0 in order to identify shared bacterial features in the plant microbiome. The different sample types were mapped on an A. wallichii illustration to better illustrate the localization of the analyzed samples in the plant. Edges connect each feature with its origin while multiple edges indicate occurrence in different sample types. Node colors indicate the assignment to distinct bacterial phyla and their size correlates with the abundance of each feature

ecosystems, especially under stress conditions [44]. In previous studies, a generally lower evenness was observed for plant microbiomes [14, 45], even if amendments that can increase the microbial community evenness were used [46]. We hypothesize that the observed evenness of the A. wallichii microbiome mainly results from the specific environmental conditions on the mountain plateau in combination with the large natural monoculture that has facilitated co-adaption of microbial communities with their plant host. Therefore, A. wallichii is also potentially interesting as a model plant to study the development and maintenance of evenly distributed bacterial communities for higher resilience against biotic as well as abiotic stress factors.

\section{Conclusions}

In the present study, we could show that $A$. wallichii harbors a spatially structured microbiome that showed specific signatures in each tissue type. A. wallichii showed special characteristics in its microbiome that make it distinguishable from other plants that were subjected to analogous analyses in the past. Furthermore, the bacterial genus Pseudomonas was found to be a universal component of the plant's bacterial communities. This makes it a promising candidate for targeted isolation and cultivation experiments in the future. In summary, the obtained insights provide a reference for microbiome studies within the Amaryllidaceae plant family as well as a basis for future approaches to mine for specific bioresources in the plant's microbiota that are potentially exploitable as natural protectants of its cultivated relatives.

Supplementary Information The online version contains supplementary material available at https://doi.org/10.1007/s00248-021-01728-5.

\begin{abstract}
Abbreviations $\mathrm{NaCl}$, Sodium chloride; rpm, Revolutions per minute; min, Minute; ml, Milliliter; $\mathrm{cm}$, Centimeter; $\mathrm{NaClO}$, Sodium hypochlorite; $\mathrm{ddH}_{2} \mathrm{O}$, Double distilled water; DNA, Deoxyribonucleic acid; PCR, Polymerase chain reaction; PNA, Peptide nucleic acid; mPNA, Mitochondrial peptide nucleic acid; pPNA, Plastidial peptide nucleic acid; bp, Base pair; PCoA, Principal coordinate analysis; ANOSIM, Analyses of similarity; OTU, Operational taxonomic unit; FDR, False discovery rate; ANOVA, Analysis of variance; g, Gram; $\mathrm{pH}$, Hydrogen ion concentration; NCBI, National Center for Biotechnology; $H^{\prime}$, Shannon index; $E$, Evenness; kg, Kilogram; mg, Milligram
\end{abstract}

Acknowledgements We want to thank Haxoi Li and Yichai Chen (both from Guiyang, China) for their help during sampling and subsequent sample processing. In addition, we want to thank the students Guoru Ren and Qianfen Zheng from the Entomology Institute of Guizhou University for their help with rendering of plant pictures implemented in visualizations. We also want to thank Shangwei Li (Guiyang, China) for allowing us to use the infrastructure in the laboratories of the Entomology Institute of Guizhou University.

Author Contribution $\mathrm{XC}$ and $\mathrm{TC}$ have conceived the idea for the study and designed it. XC conducted sample processing and DNA extractions. LK conducted bioinformatic analyses under the guidance of TC. LK and TC interpreted the bioinformatic data and integrated them in the resulting visualizations. MY and GB provided information related to the sampling site, sample collection, and the plant microbiome in general. XC and TC wrote the manuscript with specific inputs from GB.

Funding Open access funding provided by Graz University of Technology. This study was supported by the National Key Research and Development Program of China (SQ2020YFE010090), the National Natural Science Foundation of China (Grant No. 32050410297, 31701836, 31960555), the Guizhou Provincial Science and Technology Program (Grant Nos. 2019-1410, 2018-1050), the Outstanding Young Scientist Program of Guizhou Province (KY2021-026), and the Program for Introducing Talents to Chinese Universities (111 Program; D20023). In addition, the study received support by the Eurasia Pacific Uninet program (EPU 14/2019). The authors gratefully acknowledge the support of K. C. Wong Education Foundation, Hong Kong, China. 
Data availability The 16S rRNA gene fragment amplicon sequencing data used in this study was deposited at the European Nucleotide Archive (ENA; https://www.ebi.ac.uk/ena) under the accession number PRJEB34098.

Code Availability Not applicable.

\section{Declarations}

Ethics Approval This article does not contain any studies with human participants or animals performed by any of the authors.

\section{Consent to Participate Not applicable.}

Consent for Publication Not applicable.

Conflict of Interest The authors declare that they have no conflict of interests.

\section{References}

1. Nakabayashi R, Sawada Y, Aoyagi M, Yamada Y, Hirai MY, Sakurai T, Kamoi T, Rowan DD, Saito K (2016) Chemical assignment of structural isomers of sulfur-containing metabolites in garlic by liquid chromatography-Fourier transform ion cyclotron resonance-mass spectrometry. J Nutr 146:397S-402S

2. Ortiz JE, Berkov S, Pigni NB, Theoduloz C, Roitman G, Tapia A, Bastida J, Feresin GE (2012) Wild Argentinian Amaryllidaceae, a new renewable source of the acetylcholinesterase inhibitor galanthamine and other alkaloids. Molecules 17:13473-13482

3. Tallini LR, Bastida J, Cortes N, Osorio EH, Theoduloz C, Schmeda-Hirschmann G (2018) Cholinesterase inhibition activity, alkaloid profiling and molecular docking of Chilean Rhodophiala (Amaryllidaceae). Molecules 23:1532

4. Wawrosch C, Malla PR, Kopp B (2001) Micropropagation of Allium wallichii Kunth, a threatened medicinal plant of Nepal. In Vitro Cell Dev-P1 37:555-557

5. Huang D, Li Q, Zhou C, Zhou S, He X (2014) Intraspecific differentiation of Allium wallichii (Amaryllidaceae) inferred from chloroplast DNA and internal transcribed spacer fragments. J Syst Evol 52:341-354

6. Wang H, Qiu Y, Li F, Song J, Zhang X, Li X (2017) Investigation and nutrition components analysis of wild chives in Hezhang County of Guizhou Province. J Plant Genet Resour 18:1137-1144

7. Iqbal J, Abbasi BA, Batool R, Mahmood T, Ali B, Khalil AT, Kanwal S, Shah SA, Ahmad R (2018) Potential phytocompounds for developing breast cancer therapeutics: nature's healing touch. Eur J Pharmacol 827:125-148

8. Bhandari J, Muhammad B, Thapa P, Shrestha BG (2017) Study of phytochemical, anti-microbial, anti-oxidant, and anti-cancer properties of Allium wallichii. BMC Complement Altern Med 17:102

9. Berg G, Rybakova D, Grube M, Koeberl M (2016) The plant microbiome explored: implications for experimental botany. J Exp Bot 67:995-1002

10. Hassani MA, Duran P, Hacquard S (2018) Microbial interactions within the plant holobiont. Microbiome 6:58

11. Bergna A, Cernava T, Rändler M, Grosch R, Zachow C, Berg G (2018) Tomato seeds preferably transmit plant beneficial endophytes. Phytobiomes J 2:183-193

12. Chen X, Krug L, Yang H, Li H, Yang M, Berg G, Cermava T (2020) Nicotiana tabacum seed endophytic communities share a common core structure and genotype-specific signatures in diverging cultivars. Comput Struct Biotechnol J 18:287-295

13. Vurukonda SS, Vardharajula S, Shrivastava M, Skz A (2016) Enhancement of drought stress tolerance in crops by plant growth promoting rhizobacteria. Microbiol Res 184:13-24

14. Gomes EA, Lana UGP, Quensen JF, de Sousa SM, Oliveira CA, Guo J, Guimarães LJM, Tiedje JM (2018) Root-associated microbiome of maize genotypes with contrasting phosphorus use efficiency. Phytobiomes J 2:129-137

15. Deepika S, Kothamasi D (2014) Soil moisture-a regulator of arbuscular mycorrhizal fungal community assembly and symbiotic phosphorus uptake. Mycorrhiza 25:67-75

16. Compant S, Samad A, Faist H, Sessitsch A (2019) A review on the plant microbiome: Ecology, functions, and emerging trends in microbial application. J Adv Res 19:29-37

17. Chen X, Pizzatti C, Bonaldi M, Erlacher A, Saracchi M, Kunova A, Berg G, Cortesi P (2016) Biological control of lettuce basal drop and host plant colonization by rhizospheric and endophytic streptomycetes. Front Microbiol 7:714

18. Hong C, Kim JU, Lee JW, Lee SW, Jo IH (2018) Diversity of bacterial endophytes in Panax ginseng and their protective effects against pathogens. 3. Biotech 8:397

19. Bungtongdee N, Sopalun K, Laosripaiboon W, Iamtham S (2019) The chemical composition, antifungal, antioxidant and antimutagenicity properties of bioactive compounds from fungal endophytes associated with Thai orchids. J Phytopathol 167:56-64

20. Berg G, Smalla K (2009) Plant species and soil type cooperatively shape the structure and function of microbial communities in the rhizosphere. FEMS Microbiol Ecol 68:1-13

21. Bulgarelli D, Rott M, Schlaeppi K, Ver Loren van Themaat E, Ahmadinejad N, Assenza F, Rauf P, Huettel B, Reinhardt R, Schmelzer E, Peplies J, Gloeckner FO, Amann R, Eickhorst T, Schulze-Lefert P (2012) Revealing structure and assembly cues for Arabidopsis root-inhabiting bacterial microbiota. Nature 488: 91-95

22. McKernan KJ, Helbert Y, Tadigotla V, McLaughlin S, Spangler J, Zhang L, Smith D (2015) Single molecule sequencing of THCA synthase reveals copy number variation in modern drug-type Cannabis sativa L., BioRxiv: 028654

23. Wassermann B, Rybakova D, Muller C, Berg G (2017) Harnessing the microbiomes of Brassica vegetables for health issues. Sci Rep 7: 17649

24. Cernava T, Erlacher A, Soh J, Sensen CW, Grube M, Berg G (2019) Enterobacteriaceae dominate the core microbiome and contribute to the resistome of arugula (Eruca sativa Mill.). Microbiome $7: 13$

25. Golubkina N, Krivenkov L, Sekara A, Vasileva V, Tallarita A, Caruso G (2020) Prospects of Arbuscular Mycorrhizal fungi utilization in production of Allium plants. Plants (Basel) 9:2

26. Yurgel SN, Abbey L, Loomer N, Gillis-Madden R, Mammoliti M (2018) Microbial communities associated with storage onion. Phytobiomes J 2:35-41

27. Thompson LR, Sanders JG, McDonald D, Amir A, Ladau J, Locey KJ, Prill RJ, Tripathi A, Gibbons SM, Ackermann G, NavasMolina JA, Janssen S, Kopylova E, Vazquez-Baeza Y, Gonzalez A, Morton JT, Mirarab S, Zech XZ, Jiang L, Haroon MF, Kanbar J, Zhu Q, Jin SS, Kosciolek T, Bokulich NA, Lefler J, Brislawn CJ, Humphrey G, Owens SM, Hampton-Marcell J, Berg-Lyons D, McKenzie V, Fierer N, Fuhrman JA, Clauset A, Stevens RL, Shade A, Pollard KS, Goodwin KD, Jansson JK, Gilbert JA, Knight R (2017) A communal catalogue reveals Earth's multiscale microbial diversity. Nature 551:457-463

28. Lundberg DS, Yourstone S, Mieczkowski P, Jones CD, Dangl JL (2013) Practical innovations for high-throughput amplicon sequencing. Nat Methods 10:999-1002 
29. Quast C, Pruesse E, Yilmaz P, Gerken J, Schweer T, Yarza P, Peplies J, Glockner FO (2013) The SILVA ribosomal RNA gene database project: improved data processing and web-based tools. Nucleic Acids Res 41:D590-D596

30. Weller DM (2007) Pseudomonas biocontrol agents of soilborne pathogens: looking back over 30 years. Phytopathology 97:250256

31. Thomashow LS, Weller DM (1988) Role of a phenazine antibiotic from Pseudomonas fluorescens in biological control of Gaeumannomyces graminis var. tritici. J Bacteriol 170:3499-3508

32. Cramer CS (2000) Breeding and genetics of Fusarium basal rot resistance in onion. Euphytica 115:159-166

33. Chowdhury SP, Hartmann A, Gao X, Borriss R (2015) Biocontrol mechanism by root-associated Bacillus amyloliquefaciens FZB42a review. Front Microbiol 6:780

34. Whipps JM, Hand P, Pink DA, Bending GD (2008) Human pathogens and phyllosphere. Adv Appl Microbiol 64:183-221

35. Chen TZ, Xue CJ, Xu WY, Zhang M, Zhou XJ, Hai S, Shu GM (2010) Soil environmental analysis and evaluation of Pinellia ternate fields in Hezhang. Lishizhen Medicine and Materia Medica Research 21:713-714

36. Zhang H, He TB, Lin CH, He GD, Gao AQ (2015) Soil property analysis of walnut and Pinellia ternate intercropping systems in Hezhang. North Hortic 1:172-176

37. Berendsen RL, Pieterse CM, Bakker PA (2012) The rhizosphere microbiome and plant health. Trends Plant Sci 17:478-486

38. Raaijmakers JM, Paulitz TC, Steinberg C, Alabouvette C, MoënneLoccoz Y (2009) The rhizosphere: a playground and battlefield for soilborne pathogens and beneficial microorganisms. Plant Soil 321: 341-361

39. Sasse J, Martinoia E, Northen T (2018) Feed your friends: do plant exudates shape the root microbiome? Trends Plant Sci 23:25-41

40. Frey-Klett P, Pierrat JC, Garbaye J (1997) Location and survival of mycorrhiza helper Pseudomonas fluorescens during establishment of ectomycorrhizal symbiosis between Laccaria bicolor and Douglas-Fir. Appl Environ Microbiol 63:139-144

41. Micallef SA, Channer S, Shiaris MP, Colon-Carmona A (2009) Plant age and genotype impact the progression of bacterial community succession in the Arabidopsis rhizosphere. Plant Signal Behav 4:777-780

42. Inceoglu O, Al-Soud WA, Salles JF, Semenov AV, van Elsas JD (2011) Comparative analysis of bacterial communities in a potato field as determined by pyrosequencing. PLoS One 6:e23321

43. Bakker M, Manter D, Sheflin A, Weir T, Vivanco J (2012) Harnessing the rhizosphere microbiome through plant breeding and agricultural management. Plant Soil 360:1-13

44. Wittebolle L, Marzorati M, Clement L, Balloi A, Daffonchio D (2009) Initial community evenness favours functionality under selective stress. Nature 458:623-626

45. Bintarti AF, Wilson JK, Quintanilla-Tornel MA, Shade A (2020) Biogeography and diversity of multi-trophic root zone microbiomes in Michigan apple orchards: analysis of rootstock, scion, and local growing region. Phytobiomes J 4:122-132

46. Gadhave KR, Devlin PF, Ebertz A, Ross A, Gange AC (2018) Soil inoculation with Bacillus spp. modifies root endophytic bacterial diversity, evenness, and community composition in a contextspecific manner. Microb Ecol 76:741-750 